\title{
Noninvasive prenatal test for the pregnancy with Turner syndrome mosaicism 45, X/47, XXX: A case report
}

\author{
Ji Hye Kim 1 , Gun Ho Lee ${ }^{2}$, Dong Hyun $\mathrm{Cha}^{1,3}$, Eun-Hae Cho ${ }^{4}$, and Yong Wook Jung ${ }^{1, *}$ \\ ${ }^{1}$ Department of Obstetrics and Gynecology, CHA Gangnam Medical Center, CHA University, Seoul, Korea \\ ${ }^{2}$ Department of Obstetrics and Gynecology, CHA Gumi Medical Center, CHA University, Gumi, Korea \\ ${ }^{3}$ Genetics Laboratory, Fertility Center of CHA Gangnam Medical Center, CHA Universtiy, Seoul, Korea \\ ${ }^{4}$ Green Cross Genome, Yongin, Korea
}

\begin{abstract}
Noninvasive prenatal test (NIPT) is a novel screening method for the diagnosis of fetal chromosomal aneuploidies. NIPT is based on technology that detects cell-free fetal DNA in maternal plasma and analyzes it with massively parallel sequencing technology to determine whether the fetus is at risk of trisomy 21, trisomy 18, trisomy 13 or sex chromosome abnormalities (SCAs). NIPT has been reported to have sensitivity of $99 \%$ and a false positive rate of less than $1 \%$ for detecting trisomy 21 and trisomy 18. Although extension of the application of NIPT to other SCAs has been attempted, there are concerns in extending NIPT to SCAs because of maternal or fetal mosaicism, undetected maternal SCAs, and multiple pregnancies. Recently, we assessed a pregnancy with the rare Turner syndrome mosaicism $45, \mathrm{X} / 47, \mathrm{XXX}$, which was reported as $45, \mathrm{X}$ with NIPT. We present the case here and briefly review the current literatures on NIPT in testing for fetal monosomy $X$. To the best of our knowledge, this is the first report of the $45, \mathrm{X} / 47, \mathrm{XXX}$ mosaicism in Korea to be reported as 45 , X by NIPT with whole genome sequencing. This case report will provide valuable information for counseling women who want to undergo NIPT.
\end{abstract}

Key words: High-throughput nucleotide sequencing, Prenatal diagnosis, Turner syndrome, Sex chromosome aberrations, Mosaicism.

\section{Introduction}

Turner syndrome is a condition in which one of the $X$ chromosomes is partly or completely missing in a female. It is a common chromosomal abnormality affecting 1 in 2,000 live female births [1]. Mosaicism 45, X/47, XXX is a rare form of Turner syndrome, which affects $1.7 \%$ of patients with Turner syndrome [2]. Although this chromosomal abnormality may present as an abnormal maternal screening test result or abnormal ultrasonography finding, this condition is not typically associated with structural abnormalities or abnormal maternal serum screening profiles. Therefore, prenatal diagnosis of Turner syndrome is complicated, and most cases are diagnosed incidentally or postnatally.

In 1997, Lo et al. [3] demonstrated that fetal cell-free DNA (cfDNA) exists in maternal plasma during pregnancy. With

Received: 6 November 2015, Revised: 9 December 2015, Accepted: 10 December 2015, Published: 31 December 2015

${ }^{*}$ Corresponding author: Yong Wook Jung, MD, PhD

Department of Obstetrics and Gynecology, CHA Gangnam Medical Center, CHA University, 569 Nonhyeon-ro, Gangnam-gu, Seoul 06135, Korea.

Tel: +82-2-3468-3000, Fax: +82-2-3468-2616, E-mail: dumbung@chamc.co.kr

Conflict of interest: The authors (Y.W.J. and D.H.C.) received research funding for this publication from Green Cross Genome, which is a institute that develops products related to noninvasive prenatal test; test that is used to identify fetal sex chromosome abnormalities.

(c) This is an open-access article distributed under the terms of the Creative Commons Attribution Non-Commercial License (http://creativecommons.org/licenses/by-nc/4.0/) which permits unrestricted non-commercial use, distribution, and reproduction in any medium, provided the original work is properly cited.

(c) Copyright 2015 by the Korean Society of Medical Genetics 
this landmark discovery, various attempts have been made to develop noninvasive prenatal screening approaches for fetal aneuploidies using maternal plasma. Recent developments in massively parallel sequencing technology and bioinformatics techniques make it possible to analyze the fetal fraction from cfDNA in maternal blood. Noninvasive prenatal test (NIPT) using maternal blood has been widely adopted as the first line screening approach for the prenatal diagnosis of fetal aneuploidy in recent years [4]. NIPT is based on various next generation sequencing techniques and presents high sensitivity and specificity in fetal trisomy 21, trisomy 18 and trisomy 13 detection [5-7].

Researchers have tried to expand the application of NIPT to the detection of sex chromosome abnormalities (SCAs). However, there are concerns about the accuracy of diagnosing SCAs with NIPT. Recently, a prospective validation study of massively parallel sequencing-based NIPT revealed that the positive predictive value (PPV) for common SCAs was only modest [8]. Lau et al. [9] evaluated the clinical performance of NIPT by reviewing 1982 consecutive cases which included 11 cases that tested positive for SCA. Of those, only $85.7 \%$ of NIPT suspected SCA were of fetal origin. In this study, we described the first case of $45, X / 47, X X X$, which was initially reported as Turner syndrome by whole genome sequencing-based NIPT. The purpose of the study was to clarify and emphasize the limitations of NIPT in screening for SCAs in prenatal test.

This study was approved by the Institutional Review Board of the CHA Gangnam Medical Center (Seoul, Korea).

\section{Case}

A 36-year-old woman, gravida 3, para 1 at 10 weeks of gestation was referred to our clinic for genetic counseling. Her first pregnancy, which occurred 16 years ago, was uncomplicated. In 2013, she visited the Fertility Center of CHA Gangnam Medical Center due to secondary infertility. Since 2013, when she was diagnosed with $46, X X$, she had undergone in vitro fertilization and experienced two ectopic pregnancies. She received methotrexate treatment and underwent laparoscopic right salpingectomy. The case study pregnancy was a spontaneous pregnancy. Although the fetus was at 9 weeks 6 days of gestation, which is earlier than usual for measuring nuchal translucency, the transvaginal sonography showed increased thickness $(6.0 \mathrm{~mm})$ of the nuchal translucency. There was an echogenic lesion on the base of the umbilical cord, which was considered to be a physiologic umbilical hernia
(Fig. 1). Considering maternal age (>35 years) and unusual sonographic features, the woman underwent chorionic villus sampling. The woman was also referred for NIPT because she wanted to know the result as soon as possible. Chorionic villus sampling was done through the transabdominal approach. Karyotyping was performed according to the standard protocol in the Genetics Laboratory, CHA Gangnam Medical Center. We adopted a whole exome sequencing-based NIPT method, which was developed by Green Cross Genome (Yongin, Korea). The method had not been commercialized yet. Briefly, $10 \mathrm{~mL}$ of maternal blood was collected in a Vacutainer tube containing $100 \mu \mathrm{L}$ of $15 \%$ ethylenediaminetetraacetic acid. The collected blood was centrifuged at $1,600 \mathrm{~g}$ for 10 minutes. Plasma was centrifuged at $16,000 \mathrm{~g}$ for 10 minutes. cfDNA was extracted from plasma samples using the QIAmp Circulating Nucleic Acid Kit (Qiagen Inc., Hilden, Germany) according to the manufacturer's instructions. A cfDNA library was constructed. We generated approximately $2.6 \times 10^{7}$ single-end reads of 72 base pairs (bp) from the library and aligned them to the Human Reference Genome (hg 19) by using the Maximal Exact Matches algorithm (ver 0.7.5) of the Burrows-Wheeler aligner. Duplicates were marked and removed using Picard (http://broadinstitute. github.io/picard/). Whole genome sequencing was conducted according to lllumina's instructions, using the single-end reads of 76 bp sequencing protocol with the lllumina Nextseq500 platform (IIlumina Inc., San Diego, CA, USA). We counted uniquely mapped reads and then calculated G-scores for each chromosome after GC content normalization.

G-ratio $=$ Number of nucleotide aligned target chromosome / Sum of nucleotide aligned specific chromosome

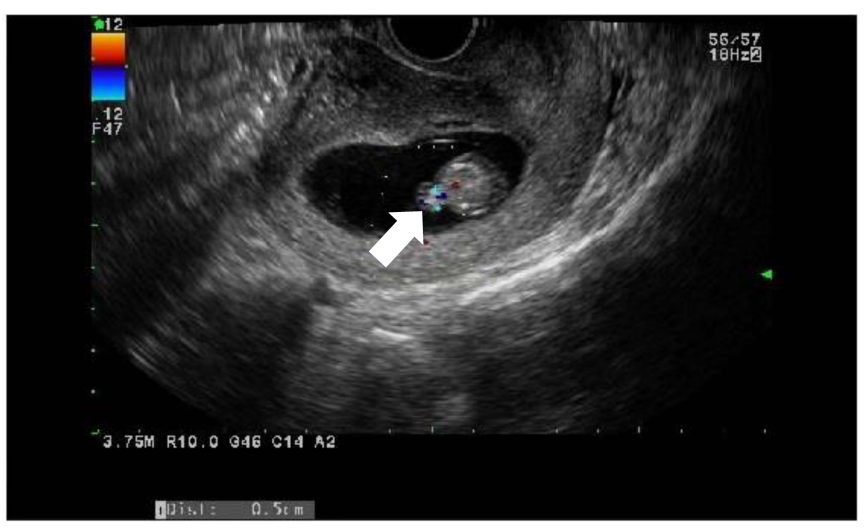

Fig. 1. Echogenic mass in the base of the umbilical cord. White arrow indicates an echogenic lesion on the base of the umbilical cord. 
G-score $=\left(\right.$ G-ratio $_{\text {sample }}-$ G-ratio mean $\left._{\text {ref }}\right) /$ G-ratio standard deviation $_{\text {ref }}$

The reference mean and standard deviation of the $G$ ratio was calculated from 101 women carrying euploid female fetuses. For our patient, the NIPT did not reveal increased risks of fetal trisomy 21 , trisomy 18 , and trisomy 13 . The fetus did not have a Y chromosome. The G-score obtained for 101 women with euploid female fetuses was -0.033 (95\% confidence interval, -1.877-1.930) for X chromosome. However, the G-score for X chromosomes of our subject was -5.5 , which showed a high probability of $45, X$ (Fig. 2A). With this result, we expected that the fetus had Turner syndrome. However, conventional karyotyping result confirmed the fetus as a mosaic of $45, \mathrm{XO}$ (mosaic ratio nearly 68\%) and 47, XXX (mosaic ratio nearly 32\%) (Fig. 2B). Preterm premature rupture of the membranes occurred at 13 weeks 3 days of gestation and resulted in stillbirth. We confirmed the fetal karyotype to be a mosaicism of $45, X / 47$, $X X X$ by using conventional cytogenetics with the abortus.

\section{Discussion}

Several studies have evaluated the performance of NIPT for chromosomal aneuploidy detection, including SCAs. Bianchi et al. [10] from the MELISSA (MatErnal BLood IS Source to Accurately Diagnose fetal aneuploidy) Study Group performed a prospective blinded study with a total of 2,882 subjects who underwent prenatal diagnostic procedures in the United States. For the diagnosis of monosomy $X$, the authors reported a sensitivity of 93.8\% (15/16) and a specificity of 99.8\% (416/417). Mazloom et al. [11] extended the application of NIPT with whole genome sequencing to detect common SCAs such as 47, XXX, $45, X, 47, X X Y$, and $47, X Y Y$. The authors performed the study in a stepwise manner with a training cohort and a validation cohort. In the training cohort, there were 8 Turner syndrome and 4 triple $X$ syndrome pregnancies. Although the NIPT that they used detected all the SCAs cases in the training cohort, only 17 out of the 21 cases of monosomy $X$ were diagnosed by NIPT in the validation cohort. There was only one case of $47, X X X$ in the validation set, and it was detected correctly. Chromosome selective sequencing was used to detect SCAs. In another study, 177 stored maternal plasma samples including 49 cases of $45, X$, 6 cases of $47, X X X, 1$ case of $47, X X Y$, and 3 cases of $47, X Y Y$, were analyzed [12]. The detection rate of $45, X$ was $91.5 \%$ (43/47). Five out of 6 cases were diagnosed correctly for $47, X X X$. With single nucleotide polymorphism (SNP)-based NIPT, SamangoSprouse et al. [13] detected $45, X$ with a sensitivity of $91.7 \%$ and a specificity of 100\% for 13 Turner syndrome samples out of 201

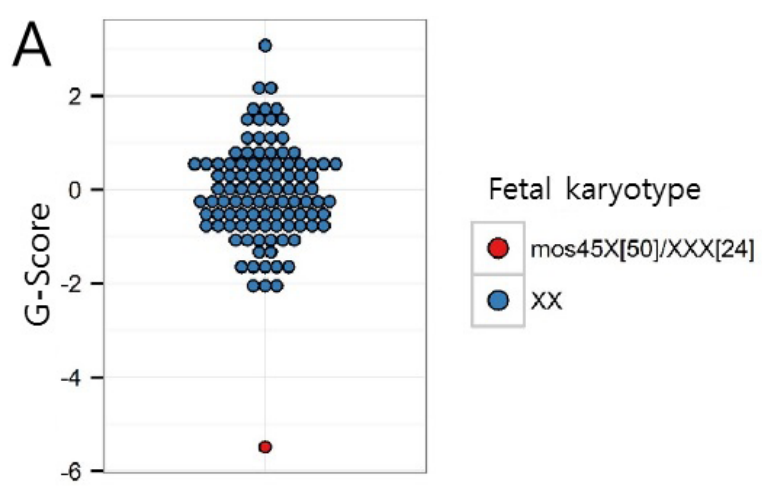

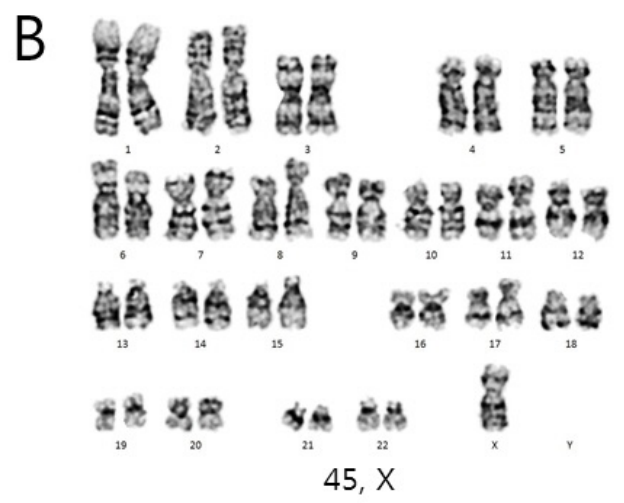

$45, x$

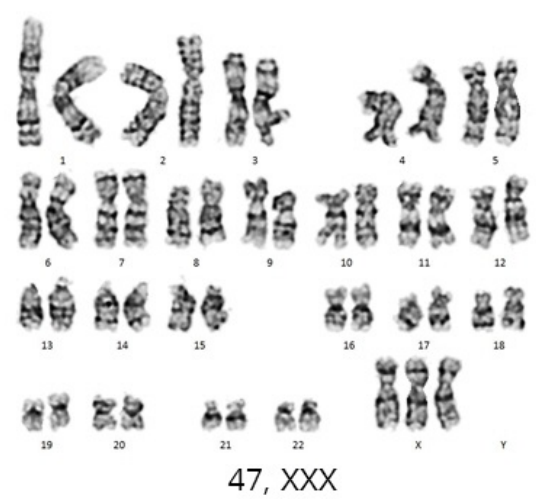

Fig. 2. (A) G-score for $45, X / 47, X X X$ showing $45, X$ test results. (B) Chromosome analysis by GTG-banding confirming 45 , $\mathrm{X} / 47, \mathrm{XXX}$ mosaicism. 
total samples. In additions, Pergament et al. [7] tested SNP-based NIPT for fetal aneuploidy and the sensitivity and specificity for monosomy X detection to be 90.0\% (9/10) and 99.9\% (953/954), respectively.

NIPT is a promising approach for the screening of fetal chromosomal abnormalities. Since it was introduced, more than half a million tests have been conducted in 61 countries [4]. Various approaches, including whole genome sequencing, targeted genome sequencing, and SNP-based sequencing have been adopted to analyze information obtained from cfDNA. Although there are advantages and disadvantages to these approaches, the accuracy and reliability are reported to be comparable among these methods. The detection sensitivity and false positive rate for trisomy 21 and trisomy 18 were reported to exceed $99 \%$ and less than 1\%, respectively [14]. A similar accuracy has been achieved for trisomy 13 as well. However, the test performance of NIPT for Turner syndrome is not as good as it is for trisomy 21 , trisomy 18 , and trisomy 13 . One of the most important effects of NIPT in the clinical setting is the decreased the fetal risk compared to that for invasive diagnostic procedures. From this point of view, a high PPV is important for a screening test. Although several researchers reported a high sensitivity of NIPT to detect SCAs as discussed above, later clinical trials have demonstrated that the PPV of NIPT is lower (about 50\%) than reported in the validation cohort. NeufeldKaiser et al. [15] reported a PPV of 55.6\% of PPVs for combined SCAs and trisomy 13 in cases over 2-year period.

There is a misconception that NIPT is diagnostic. Dar et al. [16] reported the laboratory and clinical experiences following 6 months of clinical implementation of SNP-based NIPT. During the study period, the authors encountered more than 30,000 cases and reported a $6.2 \%$ (22 cases) rate of termination without karyotype confirmation. However, there are several limitations that cause discordance between NIPT and conventional karyotyping results for the diagnosis of $45, \mathrm{X}$. The first limitation is maternal or fetal mosaicism. As a woman ages, natural loss of an $X$ chromosome may occur, which results in generation fewer $X$ chromosome fragments [17]. Mosaicism in a fetus with monosomy $X$ can be up to $50 \%$ as in case [18]. The $\mathrm{G}$-score of our subject, whose fetus was a mosaic of 45 , XO (68\%)/47, XXX (32\%), as determined by NIPT was lower than that of euploid female fetuses. However, the G-score will be affected by the ratio of mosaicism. If the mosaic ratio were 50:50, the G-score would be similar to that of euploid females, and NIPT might not show positive results for $\mathrm{X}$ chromosome abnormalities. The second limitation is maternal chromosomal abnormalities, which confound the accuracy of NIPT for the detection of fetal SCAs. Women with SCAs, for example, 47, XXX cases, are frequently asymptomatic and fertile [19]. Therefore, undiagnosed maternal SCAs might complicate test results. The third limitation is a multiple pregnancy, which may include a vanishing twin [20]. When one fetus dies in utero, cfDNA from the deceased fetus can be released into maternal circulation and, lead to discordant NIPT results. Although SNP-based NIPT has successfully identified cases of vanishing twins, further studies are required to confirm this result.

The development of NIPT is a ground-breaking advance in prenatal aneuploidy screening. NIPT is an accurate and reliable test for the autosomal trisomy screening. However, there are several limitations that need to be assessed for the detection of monosomy $X$ with NIPT based on whole exome sequencing. Here, we present a case of mosaicism $45, X / 47, X X X$ that was reported as $45, X$ by NIPT. If a clinician encounters the positive result of NIPT for sex chromosomes, then the possibility of fetal mosaicism should be discussed, and additional diagnostic tests should be considered to confirm the screening result.

\section{References}

1. Nielsen J, Wohlert M. Chromosome abnormalities found among 34,910 newborn children: results from a 13-year incidence study in Arhus, Denmark. Hum Genet 1991;87:81-3.

2. Kleczkowska $A$, Dmoch $E_{1}$ Kubien $E_{1}$ Fryns JP, Van den Berghe $H$. Cytogenetic findings in a consecutive series of 478 patients with Turner syndrome. The Leuven experience 1965-1989. Genet Couns 1990;1:227-33.

3. Lo YM, Corbetta N, Chamberlain PF, Rai V, Sargent IL, Redman CW, et al. Presence of fetal DNA in maternal plasma and serum. Lancet 1997;350:485-7

4. Chandrasekharan $S$, Minear MA, Hung A, Allyse M. Noninvasive prenatal testing goes global. Sci Transl Med 2014;6:231fs15.

5. Bianchi DW, Parker RL, Wentworth J, Madankumar R, Saffer C, Das AF, et al; CARE Study Group. DNA sequencing versus standard prenatal aneuploidy screening. N Engl J Med 2014;370:799-808.

6. Gil MM, Quezada MS, Bregant B, Ferraro M, Nicolaides KH. Implementation of maternal blood cell-free DNA testing in early screening for aneuploidies. Ultrasound Obstet Gynecol 2013;42:3440.

7. Pergament $E$, Cuckle $H$, Zimmermann $B$, Banjevic M, Sigurjonsson $S$, Ryan $A$, et al. Single-nucleotide polymorphism-based noninvasive prenatal screening in a high-risk and low-risk cohort. Obstet Gynecol 2014;124:210-8. 
8. Porreco RP, Garite TJ, Maurel K, Marusiak B; Obstetrix Collaborative

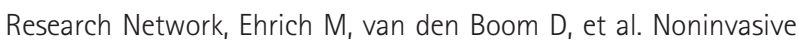
prenatal screening for fetal trisomies 21, 18, 13 and the common sex chromosome aneuploidies from maternal blood using massively parallel genomic sequencing of DNA. Am J Obstet Gynecol 2014;211: 365.e1-12.

9. Lau TK, Cheung SW, Lo PS, Pursley AN, Chan MK, Jiang F, et al. Noninvasive prenatal testing for fetal chromosomal abnormalities by low-coverage whole-genome sequencing of maternal plasma DNA: review of 1982 consecutive cases in a single center. Ultrasound Obstet Gynecol 2014;43:254-64.

10. Bianchi DW, Platt LD, Goldberg JD, Abuhamad AZ, Sehnert AJ, Rava RP; MatErnal BLood IS Source to Accurately diagnose fetal aneuploidy (MELISSA) Study Group. Genome-wide fetal aneuploidy detection by maternal plasma DNA sequencing. Obstet Gynecol 2012;119:890-901.

11. Mazloom AR, Džakula Ž, Oeth $P$, Wang $H$, Jensen $T$, Tynan J, et al. Noninvasive prenatal detection of sex chromosomal aneuploidies by sequencing circulating cell-free DNA from maternal plasma. Prenat Diagn 2013;33:591-7.

12. Nicolaides KH, Musci TJ, Struble CA, Syngelaki A, Gil MM. Assessment of fetal sex chromosome aneuploidy using directed cell-free DNA analysis. Fetal Diagn Ther 2014;35:1-6.

13. Samango-Sprouse $C$, Banjevic M, Ryan A, Sigurjonsson $S$,
Zimmermann B, Hill M, et al. SNP-based non-invasive prenatal testing detects sex chromosome aneuploidies with high accuracy. Prenat Diagn 2013;33:643-9.

14. Benn P. Non-invasive prenatal testing using cell free DNA in maternal plasma: Recent developments and future prospects. J Clin Med 2014; 3:537-65.

15. Neufeld-Kaiser WA, Cheng EY, Liu YJ. Positive predictive value of noninvasive prenatal screening for fetal chromosome disorders using cell-free DNA in maternal serum: independent clinical experience of a tertiary referral center. BMC Med 2015;13:129.

16. Dar $P$, Curnow KJ, Gross SJ, Hall MP, Stosic M, Demko $Z$, et al. Clinical experience and follow-up with large scale single-nucleotide polymorphism-based noninvasive prenatal aneuploidy testing. Am J Obstet Gynecol 2014;211:527.e1-527.e17.

17. Russell LM, Strike $P$, Browne $C E_{1}$ Jacobs PA. $X$ chromosome loss and ageing. Cytogenet Genome Res 2007;116:181-5.

18. Sybert VP, McCauley E. Turner's syndrome. N Engl J Med 2004;351: 1227-38.

19. Otter M, Schrander-Stumpel CT, Curfs LM. Triple $X$ syndrome: a review of the literature. Eur J Hum Genet 2010;18:265-71.

20. Grömminger $S$, Yagmur E, Erkan S, Nagy S, Schöck U, Bonnet J, et al. Fetal aneuploidy detection by cell-free DNA sequencing for multiple pregnancies and quality issues with vanishing twins. J Clin Med 2014; 3:679-92. 